\title{
Spatial dynamics of a vegetation model in an arid flat environment
}

\author{
Gui-Quan Sun • Li Li · Zi-Ke Zhang
}

\begin{abstract}
Self-organized vegetation patterns in space were found in arid and semi-arid areas. In this paper, we modelled a vegetation model in an arid flat environment using reaction-diffusion form and investigated the pattern formation. By using Hopf and Turing bifurcation theory, we obtain Turing region in parameters space. It is found that there are different types of stationary patterns including spotted, mixed, and stripe patterns by amplitude equation. Moreover, we discuss the changes of the wavelength with respect to biological parameters. Specifically, the wavelength be-
\end{abstract}

\section{G.-Q. Sun $(\bowtie) \cdot$ Z.-K. Zhang}

Institute of Information Economy, Hangzhou Normal University, Hangzhou 310036, People's Republic of China e-mail: gquansun@yahoo.com.cn

\section{G.-Q. Sun}

Department of Mathematics, North University of China,

Taiyuan, Shan'xi 030051, People's Republic of China

\section{Li}

Department of Mathematics, Taiyuan Institute

of Technology, Taiyuan, Shan'xi 030008,

People's Republic of China

\section{Z.-K. Zhang}

Web Sciences Center, University of Electronic Science and Technology of China, Chengdu, Sichuan 610054, People's Republic of China

\section{Z.-K. Zhang}

Department of Physics, University of Fribourg,

Chemin du Musée 3, 1700 Fribourg, Switzerland comes smaller as rainfall increases and larger as plant morality increases. The results may well explain the vegetation pattern observed in the real world and provide some new insights on preventing from desertification.

Keywords Vegetation model - Bifurcation · Pattern · Wavelength · Desertification

\section{Introduction}

Vegetation plays a critical role in the real life. On one hand, it can absorb carbon dioxide and release oxygen, which keeps the balance of the ecosystem. One the other hand, it can evaporate soil moisture into the atmosphere, which can promote global water circulation cycle. In a word, vegetation is able to keep different kinds of plants, animals, and microbes and provide all kinds of important renewable natural resources.

Spatial self-organized vegetation patterns were found by scientists in different areas [1, 2]. In arid ecosystems, regular vegetation patterns are in the form of stripes, spots, labyrinths, gaps, and so on [3-8]. The mechanisms of these patterns are different, including long-distance negative feedback, short-distance positive feedback, long-range competition, and shortrange facilitation. Moreover, in some regions, it was found that vegetation follows a power law distribution, which may be recognized as a warning signal for 
the onset of desertification [9-11]. As a result, investigation on vegetation pattern in both space and time is very important.

Since that long time data of the vegetation density is needed, it is difficult to obtain the properties of vegetation pattern in both space and time. Thus, it may provide useful information by constructing suitable mathematical models to explain the phenomenon observation in the real world. Marinov et al. presented a detailed analysis on a vegetation pattern formation model governed by a nonlinear parabolic system [12]. Ursino used a simple model to reveal the interrelations between plant ecology and hydrology, and the interplay between above and below ground mass patterns [13]. Kefi et al. investigated bistability and regular spatial patterns based on a model of vegetation dynamics in arid ecosystems [14]. Sheffer et al. posed a theory on the role of physical templates and self-organisation for vegetation patchiness [15]. Sherratt demonstrated that there were history-dependent patterns in a vegetation model [16]. Borgogno et al. reviewed mathematical models of vegetation pattern formation in ecohydrology [17]. Inspired by Klausmeier's model [1], we will study a vegetation model in an arid flat environment using reaction-diffusion equations.

By using the standard multiple-scale analysis, we can show pattern selection of reaction-diffusion equations, where the control parameter(s) and the derivatives are expanded with respect to a small parameter $\varepsilon$ and the Fredholm solubility condition is used $[18,19]$. In the small neighborhood of the critical bifurcation (Hopf or Turing) point, the critical amplitudes $A_{j}(j=1,2,3)$ follow the normal forms. Their general forms can be derived from the standard techniques of symmetry-breaking bifurcations.

The structure of this paper is as follows. In Sect. 2, we obtain a spatial vegetation model, and describe the biological meanings of these model parameters. In Sect. 3, we analyze the spatial model and derive the mathematical expression for Hopf bifurcations and Turing bifurcation critical line with respect to these parameters. On the basis of the two lines, we gain the exact Turing pattern region within the parameter space. In Sect. 4, we obtain the amplitude equations of vegetation model and by performing a series of simulations, we demonstrate the emergence of stationary patterns and interpret the meanings in
Sect. 5. Finally, some conclusions and discussion are given.

\section{Mathematical modeling and analysis}

\subsection{Model formulation}

Klausmeier proposed a very simple partial differential equation model, with equations for surface water $W(X, Y, T)$ and vegetation $U(X, Y, T)[1]$ :

$$
\begin{aligned}
& \frac{\partial W}{\partial T}=d-e W-f U^{2} W+g \nabla W \\
& \frac{\partial U}{\partial T}=a U^{2} W-b U+c \triangle U
\end{aligned}
$$

where $a$ is plant growth rate, $b$ is plant loss rate, $d$ is the rainfall, $\mathrm{e}$ is the evaporation rate of the water, and $f$ is uptake rate of plant. $\triangle=\partial^{2} / \partial X^{2}+\partial^{2} / \partial Y^{2}$ is the Laplacian operator in two-dimensional space and $\nabla=\partial / \partial X$ is a flow term.

Klausmeier's model was used to describe the vegetation pattern in a hill. However, in this study, we want to investigate the vegetation pattern formation in an arid flat environment. To this end, $\nabla W$ will be replaced by $\triangle W$ [20-22].

In order to minimize the number of parameters involved in the model system it is extremely useful to write the system in non-dimensionalized form. Although there is no unique method of doing this, it is often a good idea to relate the variables to some key relevant parameters. Thus, following Sherratt [23, 24], and taking

$w=W \frac{a}{\sqrt{e f}}, \quad u=U \sqrt{\frac{f}{e}}, \quad t=T e$,

$x=X \sqrt{\frac{e}{c}}, \quad y=Y \sqrt{\frac{e}{c}}$,

we arrive at the following equations containing dimensionless quantities:

$$
\begin{aligned}
& \frac{\partial w}{\partial t}=S-w-u^{2} w+D \Delta w, \\
& \frac{\partial u}{\partial t}=u^{2} w-B u+\triangle u,
\end{aligned}
$$

where $S=a d e^{3 / 2} / \sqrt{f}, B=b / e$ and $D=g / c$. 
The initial conditions and boundary conditions are as follows:

$\left\{\begin{array}{l}w(x, y, 0)>0, u(x, y, 0)>0, \\ (x, y) \in \Omega=\left[O, L_{x}\right] \times\left[O, L_{y}\right], \\ \frac{\partial w}{\partial \mathbf{n}}=\frac{\partial u}{\partial \mathbf{n}}=0,(x, y) \in \partial \Omega,\end{array}\right.$

where $L_{x}$ and $L_{y}$ give the size of the system in the directions of $x$ and $y$, respectively; $\mathbf{n}$ is the outward unit normal vector of the boundary $\partial \Omega$ and we assume $\partial \Omega$ is smooth. The main reason for choosing such boundary conditions is that we are interested in the selforganization of the pattern. And Neumann boundary conditions imply that there are no fluxes of populations through the boundary, i.e., no external input is imposed from outside [25-31].

\subsection{Bifurcation analysis}

In the absence of diffusion, system (2a), (2b) is corresponding to the following system:

$\frac{d w}{d t}=S-w-u^{2} w=f(u, w)$,

$\frac{d u}{d t}=u^{2} w-B u=g(u, w)$.

Note that $(w, u)$ is an equilibrium point of system (3) if it satisfies $f(u, w)=0$ and $g(u, w)=0$. When $S<2 B$, system (3) has one equilibrium: $(S, 0)$. When $S \geq 2 B$, system (3) has two equilibrium solutions in the positive quadrant. One equilibrium point, given by $\left(\left(S+\sqrt{S^{2}-4 B^{2}}\right) / 2,2 B /\left(S+\sqrt{S^{2}-4 B^{2}}\right)\right)$, which is unstable. Another equilibrium point is given by $E^{*}=\left(w^{*}, u^{*}\right)$, where

$w^{*}=\frac{S-\sqrt{S^{2}-4 B^{2}}}{2}, \quad u^{*}=\frac{2 B}{S-\sqrt{S^{2}-4 B^{2}}}$.

From the biological perspective, we are interested in studying the stability behavior of the interior equilibrium point $E^{*}$. The Jacobian matrix corresponding to this equilibrium point is as follows:

$\mathbf{J}=\left(\begin{array}{ll}a_{11} & a_{12} \\ a_{21} & a_{22}\end{array}\right)$

where

$a_{11}=-\frac{2 S}{S-\sqrt{S^{2}-4 B^{2}}}$, $a_{12}=-2 B$,

$a_{21}=\frac{4 B^{2}}{\left(S-\sqrt{S^{2}-4 B^{2}}\right)^{2}}$,

$a_{22}=B$.

Turing condition is the one in which the uniform steady state of the reaction-diffusion system is stable for the corresponding ordinary differential equations, but it is unstable in the partial differential equations with diffusion terms. First of all, we address the temporal stability of the uniform state to non-uniform perturbations [32, 33]

$\left(\begin{array}{l}w \\ u\end{array}\right)=\left(\begin{array}{l}w^{*} \\ u^{*}\end{array}\right)+\varepsilon\left(\begin{array}{l}w_{\kappa} \\ u_{\kappa}\end{array}\right) e^{\lambda t+i \kappa \vec{r}}+$ c.c. $+O\left(\varepsilon^{2}\right)$,

where $\lambda$ is the growth rate of perturbations in time $t$, $i$ is the imaginary unit and $i^{2}=-1, \kappa$ is the wavenumber, $\vec{r}=(x, y)$ is the spatial vector in two dimensions and c.c. stands for the complex conjugate. The linear instability $(\varepsilon \ll 1)$ of the uniform state is deduced from the dispersion relations. After substituting Eq. (6) into Eqs. (2a), (2b) one finds the characteristic equation for the growth rate $\lambda$ as the determinant $\operatorname{det}(\mathbf{H})$, where

$\mathbf{H}=\left(\begin{array}{cc}a_{11}-D \kappa^{2}-\lambda & a_{12} \\ a_{21} & a_{22}-\kappa^{2}-\lambda\end{array}\right)$.

Then we can obtain the eigenvalues $\lambda_{\kappa}$ as follows:

$\lambda_{\kappa}=\frac{\operatorname{tr}_{\kappa} \pm \sqrt{\left(t r_{\kappa}\right)^{2}-4 \Delta_{\kappa}}}{2}$,

where

$$
\begin{aligned}
\operatorname{tr}_{\kappa} & =a_{11}+a_{22}-\kappa^{2}(1+D) \\
& =\operatorname{tr}_{\mathbf{J}}-\kappa^{2}(1+D), \\
\Delta_{\kappa} & =a_{11} a_{22}-a_{12} a_{21}-\kappa^{2}\left(a_{11}+a_{22} D\right)+\kappa^{4} D \\
& =\Delta_{\mathbf{J}}-\kappa^{2}\left(a_{11}+a_{22} D\right)+\kappa^{4} D .
\end{aligned}
$$

Hopf bifurcation occurs when

$\operatorname{Im}\left(\lambda_{\kappa}\right) \neq 0, \quad \operatorname{Re}\left(\lambda_{\kappa}\right)=0$

at $\kappa=0$, i.e., $a_{11}+a_{22}=0$. 
Then we can get the critical value of the Hopf bifurcation parameter, $S$ :

$$
S_{H}=\frac{B^{2}}{\sqrt{B-1}} .
$$

Turing bifurcation occurs when

$\operatorname{Im}\left(\lambda_{\kappa}\right)=0, \quad \operatorname{Re}\left(\lambda_{\kappa}\right)=0, \quad$ at $\kappa=\kappa_{T} \neq 0$,

and the wavenumber $\kappa_{T}$ satisfies

$\kappa_{T}^{2}=\sqrt{\frac{\Delta_{\mathbf{J}}}{D}}$.

Then we can get the critical value of the Turing bifurcation parameter, $S$ :

$S_{T}=\frac{\sqrt{-4 B C D+3 B^{3} D^{3}-2 B^{2} C D^{2}+7 B^{2} D^{2}+8 B D B}}{B D+1}$.

where $C=\sqrt{2 B^{2} D^{2}-2 B D}$.

In [20], the diffusion coefficient values are chosen as

$g=100 m^{2} d, \quad c=0.1 m^{2} d$,

and thus we have $D=1000$. In Fig. 1, we give the dynamical regions in parameters space spanned by the parameters $S$ and $B$. The domain located below all two-bifurcations' lines, corresponds to systems with homogeneous equilibria, which is unconditionally stable. The equilibria that can be found in the area, marked by "Turing region", are stable with respect to homogeneous perturbations, but lose their stability with respect to perturbations of specific wavenumbers $\kappa$. In this region, stationary inhomogeneous patterns can be observed. In the rest, both Hopf and Turing instabilities occur. For overall analysis, we fix $B=3$ and show the real part of the eigenvalue as $S$ increases, which can be seen in Fig. 2.

\section{Amplitude equations}

The standard multiple-scale analysis yields the wellknown amplitude equations [19, 34, 35]. Close to the onset $S=S_{T}$, the eigenvalues associated to the critical modes are close to zero, and they are slowly varying modes, whereas the off-critical modes relax quickly,

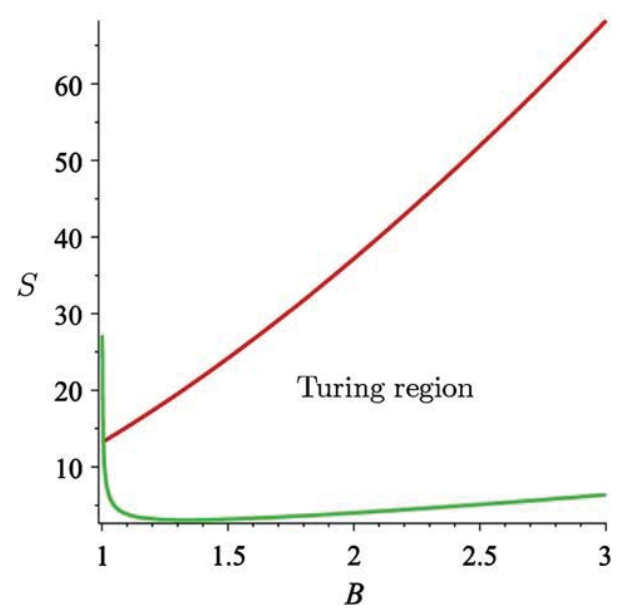

Fig. 1 Bifurcation diagram of system (2a), (2b). Turing region is bounded by the Turing bifurcation line (the blue (upper) line) and the Hopf bifurcation line (the red (lower) line) (Color figure online)

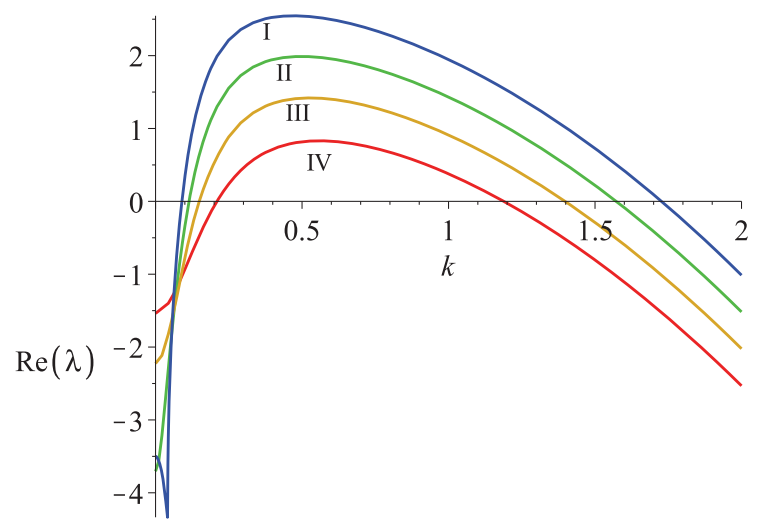

Fig. 2 The real part of the eigenvalue as $S$ increases. I: $S=10$; II: $S=30$; III: $S=40$; IV: $S=50$. This figure shows that when there is no space, the equilibrium point $E^{*}$ is stable. When combined with space, loss of stability occurs in relation to the wave numbers (Color figure online)

so only perturbations with $k$ around $k_{T}$ need considering. The whole dynamics can be therefore reduced to the dynamics of the active slow modes. The stability and the selection of the different patterns close to onset can be derived from the amplitude equations that govern the dynamics of these active modes. Turing patterns (e.g., hexagon and stripe patterns) are thus well described by a system of three active resonant pairs of modes $\left(\boldsymbol{k}_{j},-\boldsymbol{k}_{j}\right)(j=1,2,3)$ making angles of $2 \pi / 3$ and $\left|\boldsymbol{k}_{j}\right|=k_{T}$. 
We can obtain the linearized form of model (2a), (2b) at the equilibrium point $E^{*}$ as follows:

$$
\left\{\begin{aligned}
\frac{\partial x}{d t}= & a_{11} x+a_{12} y-2 u^{*} x y-w^{*} y^{2}-x y^{2} \\
& +D \Delta x \\
\frac{\partial y}{d t}= & a_{21} x+a_{22} y+2 u^{*} x y+w^{*} y^{2}+x y^{2}+\Delta y .
\end{aligned}\right.
$$

Close to onset $S=S_{T}$, the solutions of model (4a), (4b) can be expanded as

$$
\boldsymbol{U}=\boldsymbol{U}_{S}+\sum_{j=1}^{3} \boldsymbol{U}_{0}\left[A_{j} \exp \left(i \boldsymbol{k}_{j} \cdot \boldsymbol{r}\right)+\bar{A}_{j} \exp \left(-i \boldsymbol{k}_{j} \cdot \boldsymbol{r}\right)\right]
$$

At the same time, the solutions of model (12) can be expanded as

$$
\boldsymbol{U}^{0}=\sum_{j=1}^{3} \boldsymbol{U}_{0}\left[A_{j} \exp \left(i \boldsymbol{k}_{j} \cdot \boldsymbol{r}\right)+\bar{A}_{j} \exp \left(-i \boldsymbol{k}_{j} \cdot \boldsymbol{r}\right)\right]
$$

where $\boldsymbol{U}_{S}$ represents the uniform steady state. $A_{j}$ and the conjugate $\bar{A}_{j}$ are the amplitudes associated with the modes $\boldsymbol{k}_{j}$ and $-\boldsymbol{k}_{j}$, respectively. The amplitude equations are described through the equations:

$$
\left\{\begin{aligned}
\tau_{0} \frac{\partial A_{1}}{d t}= & \mu A_{1}+h \bar{A}_{2} \bar{A}_{3} \\
& -\left(g_{1}\left|A_{1}\right|^{2}+g_{2}\left(\left|A_{2}\right|^{2}+\left|A_{3}\right|^{2}\right)\right) A_{1}, \\
\tau_{0} \frac{\partial A_{2}}{d t}= & \mu A_{2}+h \bar{A}_{1} \bar{A}_{3} \\
& -\left(g_{1}\left|A_{2}\right|^{2}+g_{2}\left(\left|A_{1}\right|^{2}+\left|A_{3}\right|^{2}\right)\right) A_{2}, \\
\tau_{0} \frac{\partial A_{3}}{d t}= & \mu A_{3}+h \bar{A}_{1} \bar{A}_{2} \\
& -\left(g_{1}\left|A_{3}\right|^{2}+g_{2}\left(\left|A_{1}\right|^{2}+\left|A_{2}\right|^{2}\right)\right) A_{3},
\end{aligned}\right.
$$

where $\mu=\left(S_{T}-S\right) / S_{T}$ is a normalized distance to onset, $\tau_{0}$ is a typical relaxation time.

In the following, we will give the exact expressions of the coefficients $\tau_{0}, h, g_{1}$, and $g_{2}$.
Setting $\boldsymbol{X}=(x, y)^{T}, \boldsymbol{N}=\left(N_{1}, N_{2}\right)$, model (12) can be converted to the following system:

$\frac{\partial \boldsymbol{X}}{\partial t}=\boldsymbol{L} \boldsymbol{X}+\boldsymbol{N}$

where

$\boldsymbol{L}=\left(\begin{array}{cc}a_{11}+D \Delta & a_{12} \\ a_{21} & a_{22}+\Delta\end{array}\right)$,

$\boldsymbol{N}=\left(\begin{array}{l}N_{1} \\ N_{2}\end{array}\right)=\left(\begin{array}{c}-2 u^{*} x y-w^{*} y^{2}-x y^{2} \\ 2 u^{*} x y+w^{*} y^{2}+x y^{2}\end{array}\right)$.

During the calculation, we just analysis the behavior of the controlled parameter close to onset $S=S_{T}$. With this method, we can expand $S$ in the following term:

$S_{T}-S=\varepsilon S_{1}+\varepsilon^{2} S_{2}+\varepsilon^{3} S_{3}+O\left(\varepsilon^{4}\right)$,

where $\varepsilon$ is a small parameter. Expanding the variable $\boldsymbol{X}$ and the nonlinear term $\boldsymbol{N}$ according to this small parameter, we have the following results:

$$
\begin{aligned}
\boldsymbol{X} & =\left(\begin{array}{l}
x \\
y
\end{array}\right) \\
& =\varepsilon\left(\begin{array}{l}
x_{1} \\
y_{1}
\end{array}\right)+\varepsilon^{2}\left(\begin{array}{l}
x_{2} \\
y_{2}
\end{array}\right)+\varepsilon^{3}\left(\begin{array}{l}
x_{3} \\
y_{3}
\end{array}\right)+O\left(\varepsilon^{4}\right),
\end{aligned}
$$

$\boldsymbol{N}=\varepsilon^{2} \boldsymbol{h}^{2}+\varepsilon^{3} \boldsymbol{h}^{3}+O\left(\varepsilon^{4}\right)$,

where $\boldsymbol{h}^{2}$ and $\boldsymbol{h}^{3}$ are corresponding to the second and the third order of $\varepsilon$ in the expansion of the nonlinear term $\boldsymbol{N}$. At the same time, the linear operator $\boldsymbol{L}$ can be expanded as follows:

$\boldsymbol{L}=\boldsymbol{L}_{T}+\left(S_{T}-S\right) \boldsymbol{M}$,

where

$\boldsymbol{L}_{T}=\left(\begin{array}{cc}a_{11}^{*}+D \nabla^{2} & a_{12}^{*} \\ a_{21}^{*} & a_{22}^{*}+\nabla^{2}\end{array}\right)$,

$\boldsymbol{M}=\left(\begin{array}{ll}b_{11} & b_{12} \\ b_{21} & b_{22}\end{array}\right)$.

Here, one can have the expression of $a_{i j}^{*}$ by substituting $A_{T}$ for $A$ in $a_{i j}$ and $b_{i j}$ is easy to be obtained. The core of the standard multiple-scale analysis is separating the dynamical behavior of the system according to different time scale or spatial scale. We just need 
to separate the time scale for model (16) (i.e., $T_{0}=t$, $\left.T_{1}=\varepsilon t, T_{2}=\varepsilon^{2} t\right)$. Each time scale $T_{i}$ can be considered as independent variable. The derivative with respect to time becomes the following form:

$$
\frac{\partial}{\partial t}=\frac{\partial}{\partial T_{0}}+\varepsilon \frac{\partial}{\partial T_{1}}+\varepsilon^{2} \frac{\partial}{\partial T_{2}}+O\left(\varepsilon^{3}\right) .
$$

Since that amplitude $A$ is a variable that changes slowly, the derivative with respect to time $\frac{\partial}{\partial T_{0}}$, which changes fast does not have an effect upon the amplitude $A$. As a result, we have the following result:

$\frac{\partial A}{\partial t}=\varepsilon \frac{\partial A}{\partial T_{1}}+\varepsilon^{2} \frac{\partial A}{\partial T_{2}}+O\left(\varepsilon^{3}\right)$.

By using the Eqs. (18), (19), (20), and (21) and expanding Eq. (16) according to different orders of $\varepsilon$, we can obtain three equations as follows:

The first order of $\varepsilon$ :

$\boldsymbol{L}_{T}\left(\begin{array}{l}x_{1} \\ y_{1}\end{array}\right)=0$

The second order of $\varepsilon$ :

$$
\begin{aligned}
\boldsymbol{L}_{T}\left(\begin{array}{l}
x_{2} \\
y_{2}
\end{array}\right)= & \frac{\partial}{\partial T_{1}}\left(\begin{array}{l}
x_{1} \\
y_{1}
\end{array}\right)-S_{1} \boldsymbol{M}\left(\begin{array}{l}
x_{1} \\
y_{1}
\end{array}\right) \\
& -\left(\begin{array}{c}
-2 u^{*} x_{1} y_{1}-w^{*} y_{1}^{2} \\
2 u^{*} x_{1} y_{1}+w^{*} y_{1}^{2}
\end{array}\right) .
\end{aligned}
$$

The third order of $\varepsilon$ :

$$
\begin{aligned}
\boldsymbol{L}_{T}\left(\begin{array}{l}
x_{3} \\
y_{3}
\end{array}\right)= & \frac{\partial}{\partial T_{1}}\left(\begin{array}{l}
x_{2} \\
y_{2}
\end{array}\right)+\frac{\partial}{\partial T_{2}}\left(\begin{array}{l}
x_{1} \\
y_{1}
\end{array}\right) \\
& -S_{1} \boldsymbol{M}\left(\begin{array}{l}
x_{2} \\
y_{2}
\end{array}\right)-S_{2} \boldsymbol{M}\left(\begin{array}{l}
x_{1} \\
y_{1}
\end{array}\right)-Z,
\end{aligned}
$$

where

$$
Z=\left(\begin{array}{c}
-2 u^{*}\left(x_{1} y_{2}+y_{1} x_{2}\right)-2 w^{*} y_{1} y_{2}-x_{1} y_{1}^{2} \\
2 u^{*}\left(x_{1} y_{2}+y_{1} x_{2}\right)+2 w^{*} y_{1} y_{2}+x_{1} y_{1}^{2}
\end{array}\right) .
$$

For the first order of $\varepsilon$ :

$\boldsymbol{L}_{T}\left(\begin{array}{l}x_{1} \\ y_{1}\end{array}\right)=0$

As $\boldsymbol{L}_{T}$ is the linear operator of the system close to the onset, $\left(x_{1}, y_{1}\right)^{T}$ is the linear combination of the eigenvectors that corresponds to the eigenvalue 0 . Solving the first order of $\varepsilon$, we have

$$
\begin{aligned}
\left(\begin{array}{l}
x_{1} \\
y_{1}
\end{array}\right)= & \left(\begin{array}{c}
\frac{a_{11}^{*}-a_{22}^{*} D}{2 a_{21}^{*} D} \\
1
\end{array}\right)\left(W_{1} \exp \left(i \boldsymbol{k}_{1} \boldsymbol{r}\right)\right. \\
& \left.+W_{2} \exp \left(i \boldsymbol{k}_{2} \boldsymbol{r}\right)+W_{3} \exp \left(i \boldsymbol{k}_{3} \boldsymbol{r}\right)\right) \\
& + \text { c.c. },
\end{aligned}
$$

where $\left|\boldsymbol{k}_{j}\right|=k_{T}^{*}, W_{j}$ is the amplitude of the mode $\exp \left(i \boldsymbol{k}_{j} \boldsymbol{r}\right)$ when the system is under the first order perturbation.

For the second order of $\varepsilon$, we have

$$
\begin{aligned}
\boldsymbol{L}_{T}\left(\begin{array}{l}
x_{2} \\
y_{2}
\end{array}\right)= & \frac{\partial}{\partial T_{1}}\left(\begin{array}{l}
x_{1} \\
y_{1}
\end{array}\right)-S_{1} M\left(\begin{array}{l}
x_{1} \\
y_{1}
\end{array}\right) \\
& -\left(\begin{array}{c}
-2 u^{*} x_{1} y_{1}-w^{*} y_{1}^{2} \\
2 u^{*} x_{1} y_{1}+w^{*} y_{1}^{2}
\end{array}\right) \\
= & \left(\begin{array}{l}
F_{x} \\
F_{y}
\end{array}\right) .
\end{aligned}
$$

According to the Fredholm solubility condition, the vector function of the right hand of Eq. (25) must be orthogonal with the zero eigenvectors of operator $\boldsymbol{L}_{c}^{+}$. $\boldsymbol{L}_{c}^{+}$is the adjoint operator of $\boldsymbol{L}_{c}$. In this system, the zero eigenvectors of operator $\boldsymbol{L}_{c}^{+}$are

$$
\left(\begin{array}{c}
1 \\
-\frac{a_{11}^{*}-a_{22}^{*} D}{2 a_{21}^{*}}
\end{array}\right) \exp \left(-i \boldsymbol{k}_{j} \boldsymbol{r}\right)+\text { c.c. } \quad(j=1,2,3) .
$$

The orthogonality condition is

$$
\left(1,-\frac{a_{11}^{*}-a_{22}^{*} D}{2 a_{21}^{*}}\right)\left(\begin{array}{c}
F_{x}^{i} \\
F_{y}^{i}
\end{array}\right)=0
$$

where $F_{x}^{i}$ and $F_{y}^{i}$, separately, represent the coefficients corresponding to $\exp \left(i \boldsymbol{k}_{j} \boldsymbol{r}\right)$ in $F_{x}$ and $F_{y}$. Taking $\exp \left(i \boldsymbol{k}_{1} \boldsymbol{r}\right)$, for example, we have

$$
\begin{aligned}
(1-D) \frac{\partial W_{1}}{\partial T_{1}} \\
=S_{1}\left(l b_{11}+b_{12}-D l\left(b_{21}+b_{22}\right)\right) W_{1} \\
\quad-(1+D l)\left(4 l S_{T} u^{*}+2 l S_{T} w^{*}\right) \bar{W}_{2} \bar{W}_{3} .
\end{aligned}
$$


The coefficients in Eq. (28) are obtained by solving the sets of the linear equations about $\exp (0)$, $\exp \left(i \boldsymbol{k}_{j} \boldsymbol{r}\right), \exp \left(i 2 \boldsymbol{k}_{j} \boldsymbol{r}\right), \exp \left(i\left(\boldsymbol{k}_{j}-\boldsymbol{k}_{k}\right) \boldsymbol{r}\right)$.

With this method, we have

$$
\begin{aligned}
& \left(\begin{array}{c}
X_{0} \\
Y_{0}
\end{array}\right)=\left(\begin{array}{c}
x_{0} \\
y_{0}
\end{array}\right)\left(\left|W_{1}\right|^{2}+\left|W_{2}\right|^{2}+\left|W_{3}\right|^{2}\right), \\
& X_{i}=l Y_{i}, \\
& \left(\begin{array}{c}
X_{j j} \\
Y_{j j}
\end{array}\right)=\left(\begin{array}{c}
x_{11} \\
y_{11}
\end{array}\right) W_{j}^{2}, \\
& \left(\begin{array}{c}
X_{j k} \\
Y_{j k}
\end{array}\right)=\left(\begin{array}{c}
x^{*} \\
y^{*}
\end{array}\right) W_{j} \bar{W}_{k} .
\end{aligned}
$$

For the third order of $\varepsilon$, we have

$$
\begin{aligned}
\boldsymbol{L}_{T}\left(\begin{array}{l}
x_{3} \\
y_{3}
\end{array}\right)= & \frac{\partial}{\partial T_{1}}\left(\begin{array}{l}
x_{2} \\
y_{2}
\end{array}\right)+\frac{\partial}{\partial T_{2}}\left(\begin{array}{l}
x_{1} \\
y_{1}
\end{array}\right) \\
& -S_{1} M\left(\begin{array}{l}
x_{2} \\
y_{2}
\end{array}\right)-S_{2} M\left(\begin{array}{l}
x_{1} \\
y_{1}
\end{array}\right)-Z .
\end{aligned}
$$

Using the Fredholm solubility condition again, we can obtain

$$
\begin{aligned}
(1- & D) l \frac{\partial W_{1}}{\partial T_{2}}+(1-D) l \frac{\partial Y_{1}}{\partial T_{1}} \\
= & S_{2}\left(l b_{11}+b_{12}-D l\left(l b_{21}+b_{22}\right)\right) W_{1} \\
& +S_{1}\left(l b_{11}+b_{12}-D l\left(l b_{21}+b_{22}\right)\right) Y_{1} \\
& +(1+D l)\left(4 S_{T} u^{*}+2 S_{T} w^{*}\right)\left(\bar{W}_{3} \bar{Y}_{2}+\bar{W}_{2} \bar{Y}_{3}\right) \\
& -\left(G_{1}\left|W_{1}\right|^{2}+G_{2}\left(\left|W_{2}\right|^{2}+\left|W_{3}\right|^{2}\right)\right) W_{1} .
\end{aligned}
$$

By transformation of $W$, the other two equations can be obtained and the amplitude $A_{i}$ can be expanded as

$$
A_{i}=\varepsilon W_{i}+\varepsilon^{2} V_{i}+O\left(\varepsilon^{3}\right) .
$$

For the order $\varepsilon^{2}$ and $\varepsilon^{3}$, we can obtain the amplitude equation corresponding to $A_{1}$ as follows:

$$
\begin{aligned}
\tau_{0} \frac{\partial A_{1}}{\partial t}= & \mu A_{1}+h \overline{A_{2}} \overline{A_{3}} \\
& -\left(g_{1}\left|A_{1}\right|^{2}+g_{2}\left(\left|A_{2}\right|^{2}+\left|A_{3}\right|^{2}\right)\right) A_{1},
\end{aligned}
$$

where

$$
\tau_{0}=\frac{D-1}{S_{T}\left[l b_{11}+b_{12}-D l\left(l b_{21}+b_{22}\right)\right]},
$$

$h=\frac{(1+D l)\left(4 S_{T} u^{*}+2 S_{T} w^{*}\right)}{S_{T}\left[l b_{11}+b_{12}-D l\left(l b_{21}+b_{22}\right)\right]}$,

$g_{1}=\frac{K_{1}}{S_{T}\left[l b_{11}+b_{12}-D l\left(l b_{21}+b_{22}\right)\right]}$,

and

$g_{2}=\frac{K_{2}}{S_{T}\left[l b_{11}+b_{12}-D l\left(l b_{21}+b_{22}\right)\right]}$,

with $K_{1}=(1+D)\left[\left(-2 S_{T} u^{*} l-2 S_{T} w^{*}\right)\left(y_{0}+y_{11}\right)-\right.$ $\left.2 S_{T} u^{*}\left(x_{0}+x_{11}\right)-3 S_{T} l\right]$ and $K_{2}=(1+D) \times$ $\left[\left(-2 S_{T} u^{*} l-2 S_{T} w^{*}\right)\left(y_{0}+y_{11}\right)-2 S_{T} u^{*}\left(x_{0}+x_{11}\right)-\right.$ $\left.6 S_{T} l\right]$.

The other two equations of (15) can be obtained through the transformation of the subscript of $A$. Each amplitude in Eq. (14) can be decomposed to mode $\rho_{i}=\left|A_{i}\right|$ and a corresponding phase angle $\varphi_{i}$. Then, substituting $A_{i}=\rho_{i} \exp \left(i \varphi_{i}\right)$ into Eq. (14) and separating the real and imaginary parts, we can get four differential equations of the real variables as follows [36]:

$\tau_{0} \frac{\partial \varphi}{d t}=-h \frac{\rho_{1}^{2} \rho_{2}^{2}+\rho_{1}^{2} \rho_{3}^{2}+\rho_{2}^{2} \rho_{3}^{2}}{\rho_{1} \rho_{2} \rho_{3}} \sin \varphi$,

$\tau_{0} \frac{\partial \rho_{1}}{d t}=\mu \rho_{1}+h \rho_{2} \rho_{3} \cos \varphi-g_{1} \rho_{1}^{3}-g_{2}\left(\rho_{2}^{2} \rho_{3}^{2}\right) \rho_{1}$,

$\tau_{0} \frac{\partial \rho_{2}}{d t}=\mu \rho_{2}+h \rho_{1} \rho_{3} \cos \varphi-g_{1} \rho_{2}^{3}-g_{2}\left(\rho_{1}^{2} \rho_{3}^{2}\right) \rho_{2}$,

$\tau_{0} \frac{\partial \rho_{3}}{d t}=\mu \rho_{3}+h \rho_{1} \rho_{2} \cos \varphi-g_{1} \rho_{3}^{3}-g_{2}\left(\rho_{1}^{2} \rho_{2}^{2}\right) \rho_{3}$.

where $\varphi=\varphi_{1}+\varphi_{2}+\varphi_{3}$.

System (33) may have four different types of solutions [18].

(1) The stationary state $(\mathrm{O})$, corresponding to

$$
\rho_{1}=\rho_{2}=\rho_{3}=0
$$

is stable for $\mu<\mu_{2}=0$, and unstable for $\mu>\mu_{2}$.

(2) Stripe patterns $(S)$, corresponding to

$\rho_{1}=\sqrt{\frac{\mu}{g_{1}}} \neq 0, \quad \rho_{2}=\rho_{3}=0$

are stable for $\mu>\mu_{3}=\frac{h^{2} g_{1}}{\left(g_{2}-g_{1}\right)^{2}}$, and unstable for $\mu<\mu_{3}$. 
(3) Hexagon patterns $\left(H_{0}, H_{\pi}\right)$ are corresponding to

$$
\rho_{1}=\rho_{2}=\rho_{3}=\frac{|h| \pm \sqrt{h^{2}+4\left(g_{1}+2 g_{2} \mu\right)}}{2\left(g_{1}+2 g_{2}\right)},
$$

with $\varphi=0$ or $\pi$, and exist when

$$
\mu>\mu_{1}=\frac{-h^{2}}{4\left(g_{1}+2 g_{2}\right)} .
$$

The solution $\rho^{+}=\frac{|h|+\sqrt{h^{2}+4\left(g_{1}+2 g_{2} \mu\right)}}{2\left(g_{1}+2 g_{2}\right)}$ is stable only for

$\mu<\mu_{4}=\frac{2 g_{1}+g_{2}}{\left(g_{2}-g_{1}\right)^{2}} h^{2}$,

and $\rho^{-}=\frac{|h|-\sqrt{h^{2}+4\left(g_{1}+2 g_{2} \mu\right)}}{2\left(g_{1}+2 g_{2}\right)}$ is always unstable.

(4) The mixed states are corresponding to

$\rho_{1}=\frac{|h|}{g_{2}-g_{1}}, \quad \rho_{2}=\rho_{3}=\sqrt{\frac{\mu-g_{1} \rho_{1}^{2}}{g_{1}+g_{2}}}$,

with $g_{2}>g_{1}$. They exist when $\mu>\mu_{3}$ and are always unstable.

We show the analysis results on the above in Fig. 3 with $B=3$ and $D=1000$. It is found that when $\mu=\mu_{2}, \mu_{3}$ and $\mu_{4}$, the corresponding values of $S$ are $S=6.43537822,34.11923562$, and 61.56901126 .

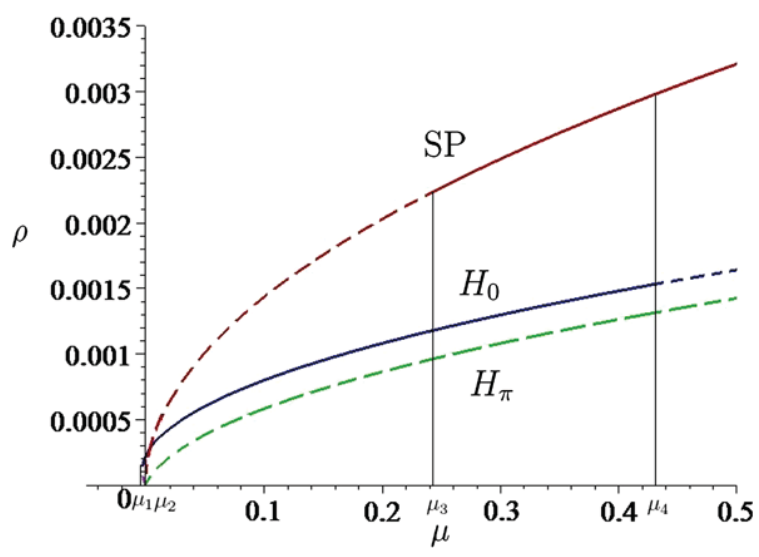

Fig. 3 Bifurcation diagram of model (2a), (2b). We set the parameter values as $B=3, D=1000 . H_{0}$ : hexagon patterns with $\varphi=0 ; H_{\pi}$ : hexagon patterns with $\varphi=\pi$; SP: stripe patterns. Solid curve: stable state; dash line: unstable state. $\mu_{1}=-0.00314651, \mu_{2}=0, \mu_{3}=0.24718849$, and $\mu_{4}=0.43681987$
When the controlled parameter $\mu$ increases to the critical point $\mu_{2}=0$, the stationary state of the system begins to lose stability. If $\mu_{1}<\mu<\mu_{2}$, then the system exists a bistable region in the range of the controlled parameter. The emergence of stripe patterns derives from supercritical bifurcation which are unstable for $\mu<\mu_{3}$ and stable for $\mu>\mu_{3}$. When the controlled parameter $\mu$ exceeds $\mu_{4}$, there is coexistence of hexagon and stripe pattern.

\section{Pattern formation}

Since the dynamical behavior of the spatial model cannot be studied by using analytical methods or normal forms, we have to perform numerical simulations by computer. The continuous problem defined by the reaction-diffusion system in two-dimensional space is solved in a discrete domain with $M \times N$ lattice sites. The space between the lattice points is defined by the lattice constant $\Delta H$. The time evolution is also discrete, i.e., the time goes in steps of $\Delta t$. The time evolution can be solved by using the Euler method. In the present paper, we set $\Delta H=1, \Delta t=0.00001$ and $M=N=150$. And it was also checked that a further decrease of the step values did not lead to any significant modification of the results.

In the following, we will perform a series of numerical simulations of the spatially extended model (2a), (2b) in two-dimensional spaces, and the qualitative results are shown by figures. We keep $B=3, D=1000$, and $S$ is regarded as a parameter. All our numerical simulations are employed with a system size of $150 \times 150$ space units. We run the simulations until they reach a stationary state or until they show a behavior that does not seem to change its characteristics anymore. In this paper, we want to know the distribution of the vegetation. As a result, we will restrict our analysis of pattern formation of variable $u$.

Figure 4 shows the evolution of the spatial pattern of vegetation at $0,10000,50000$, and 300000 iterations with $S=10$. It can be concluded from this figure that random distribution can result in regular spotted patterns. The parameter values set in Fig. 4 satisfy $\mu_{2}<\mu=0.07431124<\mu_{3}$, which means $H_{0}$ spotted pattern will emerge. That is to say, numerical results in Fig. 4 are consistent with the results shown in Fig. 3. 
(a)
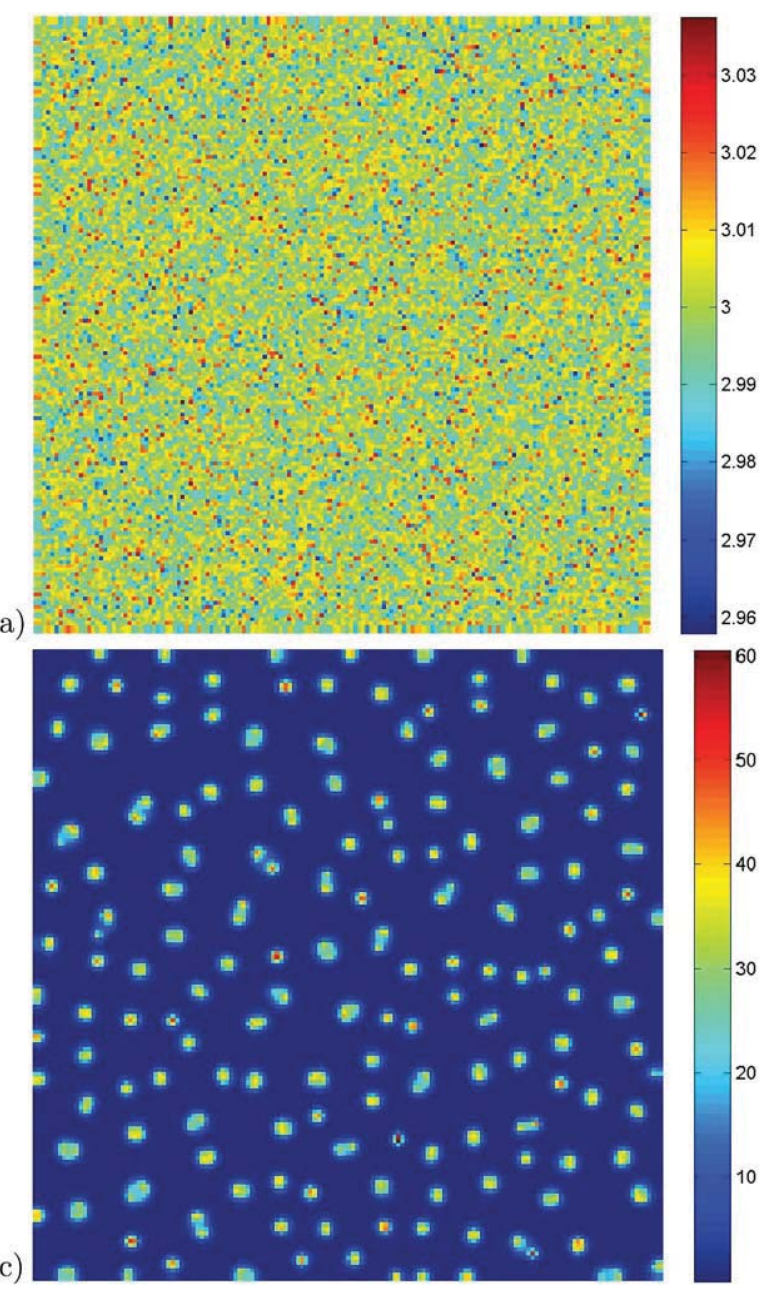

(b)

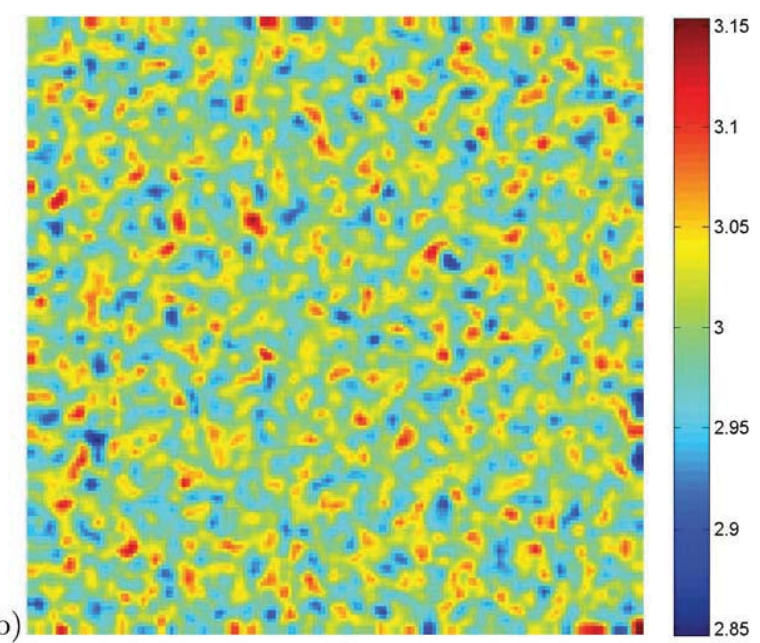

(d)

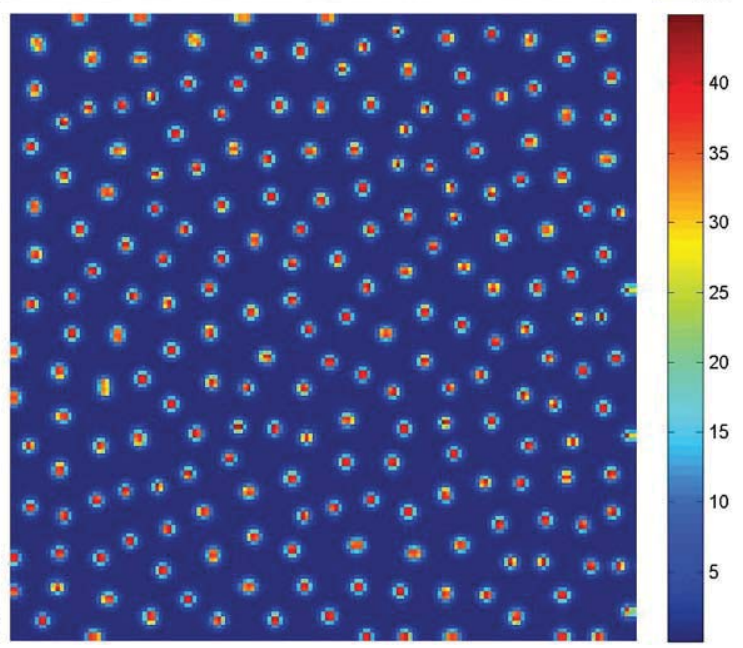

Fig. 4 Snapshots of contour pictures of the time evolution of vegetation at different instants with $S=10, D=1000$ and $B=3$. (a) 0 iteration; (b): 10000 iterations; (c) 50000 iterations; (d) 300000 iterations (Color figure online)

To well describe the evolution of the vegetation pattern, we show space-time plot in Fig. 5. The method of space-time plot is to let one direction of space be a constant (here, we set $x=75$, the center line of each snapshot) and show the evolution of pattern on the other direction of space. As a result, the space-time time plots show the evolution process of the vegetation as a function of time $t$ and space $y$. As seen from Fig. 5, it shows that Turing pattern will emerge when time is long enough.

In Fig. 6, the time series of the spatially averaged values of the vegetation density shows that in the first intervals of simulations these values change fast as time increases. One can see that at $t \approx 50$, the vegetation density reaches a constant value and the mean

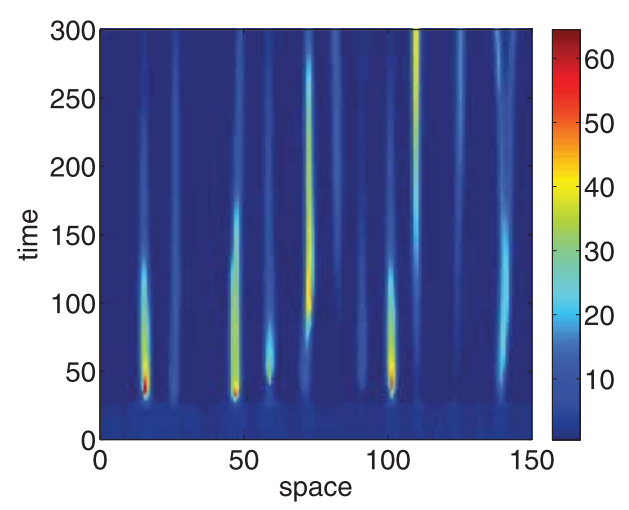

Fig. 5 Space-time plot corresponding to Fig. 4. This figure shows that there are stationary patterns for a long enough time (Color figure online) 


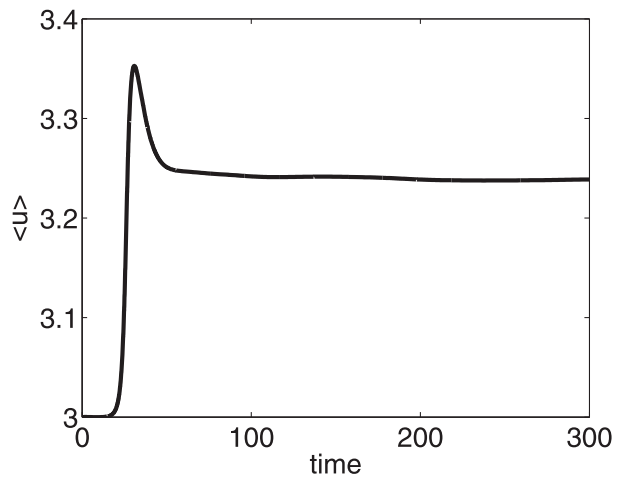

Fig. 6 The time series of the mean density of vegetation population. Parameters values are the same as Fig. 4

(a)
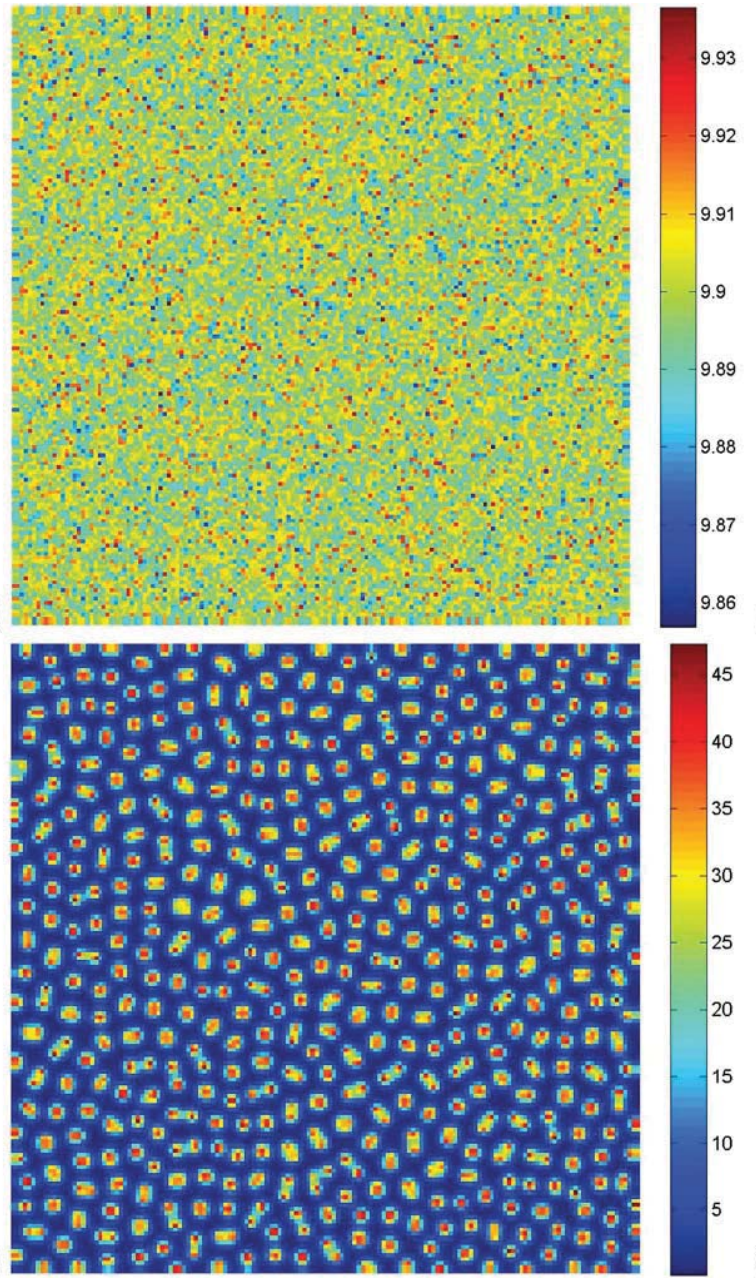

value increases slower. At this time, the vegetation pattern formation has almost completely evolved and the system has reached its steady state which means stationary patterns appear in this system.

In Fig. 7, we show the spatial pattern of vegetation at $0,30,000,100,000$, and 300,000 iterations with $S=30$ and the initial condition is the same as that in Fig. 4. It can be seen from this figure that the random initial distribution of vegetation will lead to an inconspicuous stripe shape. After these forms, spotted-like patterns will emerge and persist for a very long time, and the spotted patterns occupy the two-dimensional space. As time further increases, the pattern dynamics of the system stay the same. (b)
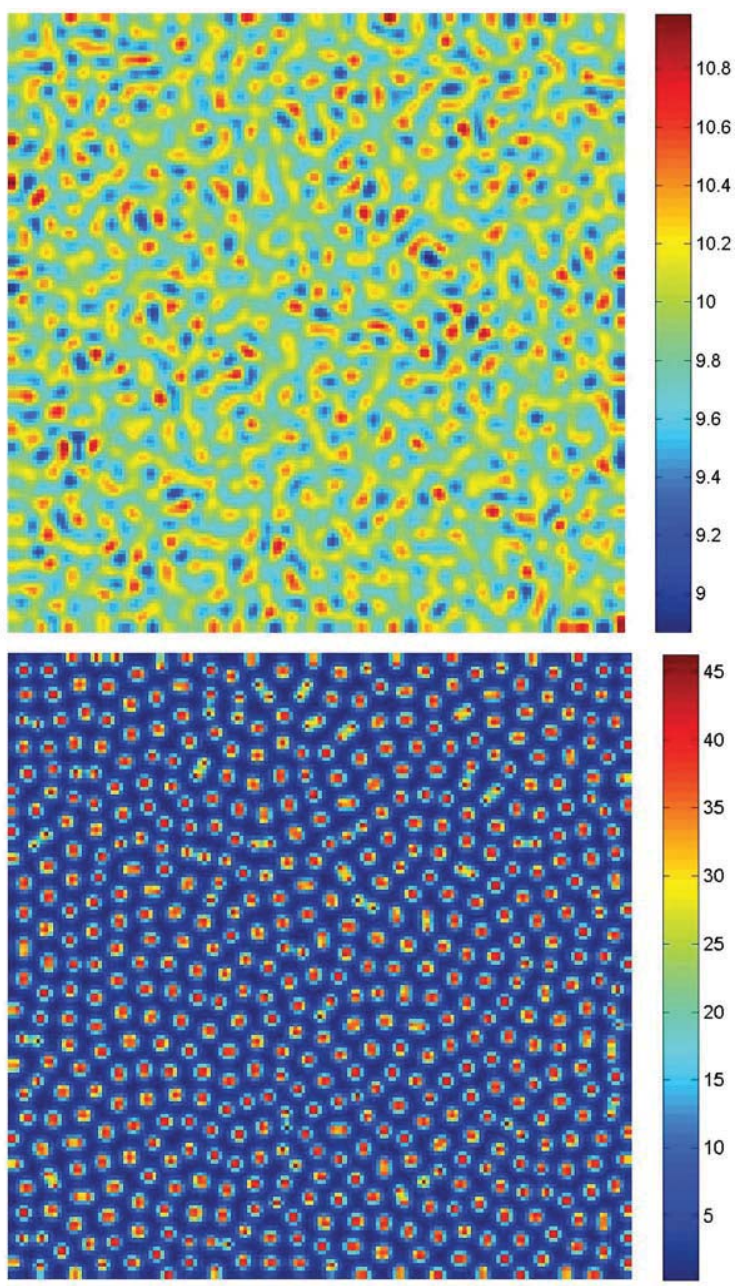

Fig. 7 Snapshots of contour pictures of the time evolution of vegetation at different instants with $S=30, D=1,000$ and $B=3$. (a) 0 iteration; (b) 30,000 iterations; (c) 100,000 iterations; (d) 300,000 iterations (Color figure online) 
(a)
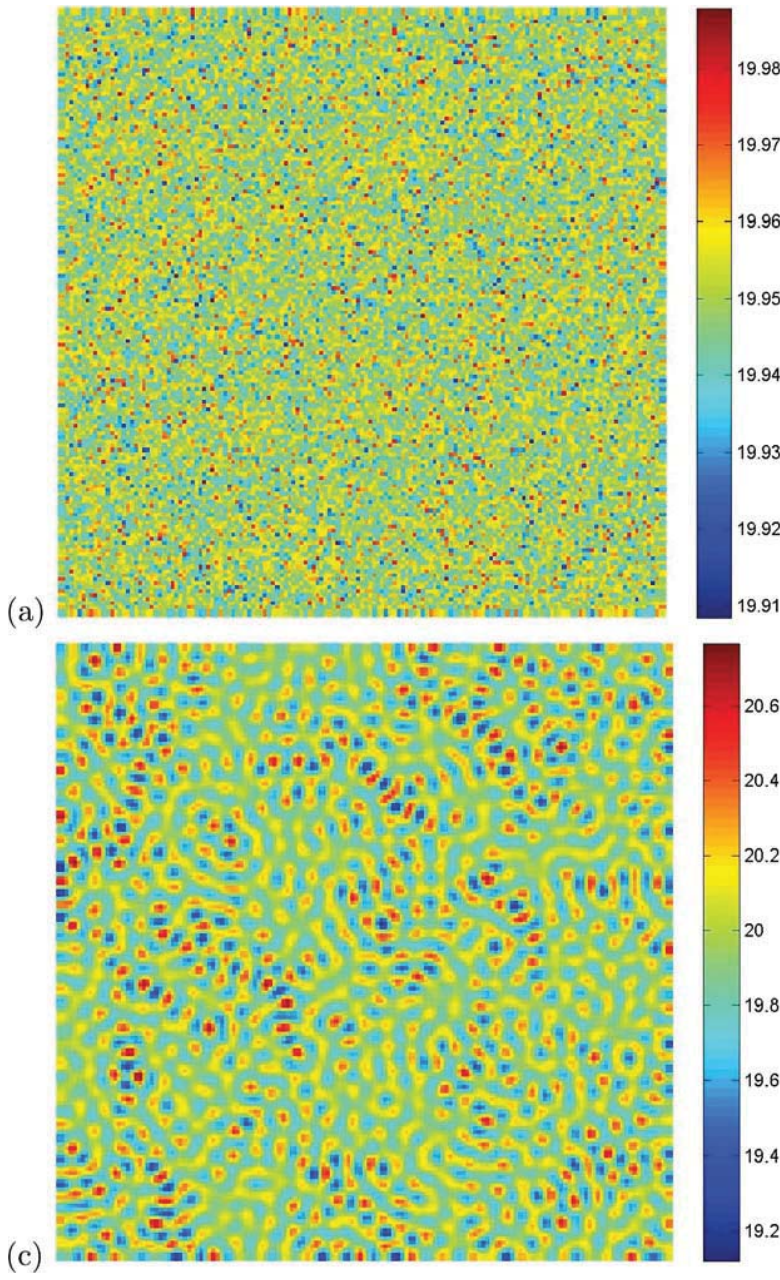

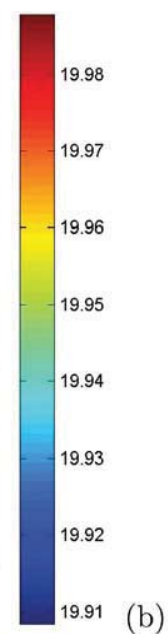

(b)

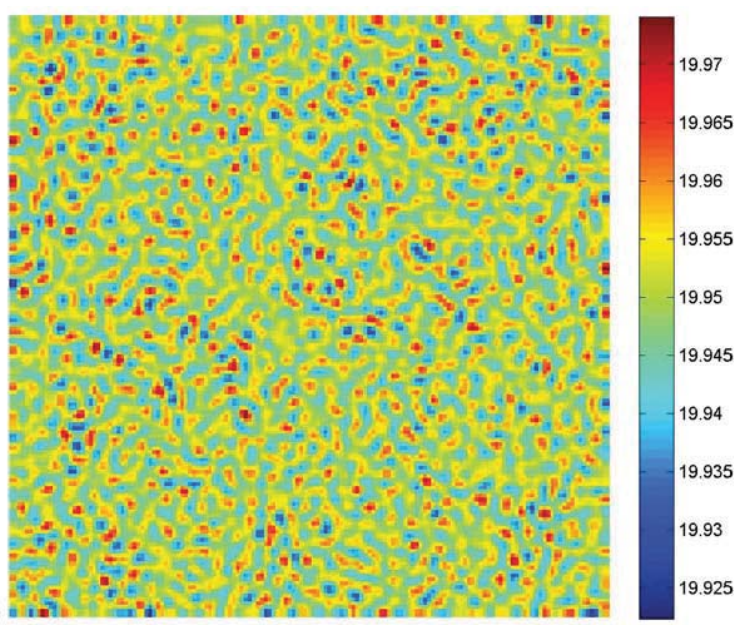

(d)

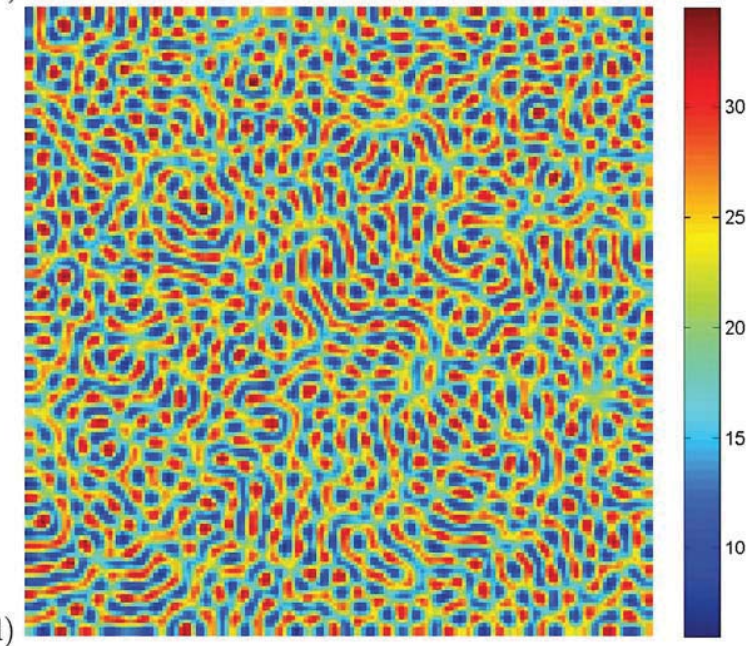

Fig. 8 Snapshots of contour pictures of the time evolution of vegetation at different instants with $S=60, D=1,000$ and $B=3$. (a) 0 iteration; (b) 20,000 iterations; (c) 150,000 iterations; (d) 300,000 iterations (Color figure online)

Compared with Figs. 4, 7 has three main differences with it. Firstly, the spotted pattern in Fig. 7 is similar with a circle. However, in Fig. 4, it looks like a square shape. Secondly, we found the time for the spotted patterns emerge in Fig. 7 is short than it in Fig. 4. Lastly, the mean density of vegetation in Fig. 7 is higher than that in Fig. 4, which means rainfall plays a positive role on vegetation growth.

In Fig. 8, we show the spatial pattern of vegetation at $0,20,000,150,000$, and 300,000 iterations with $S=60$. In that case, we obtain that $\mu=0.35425682 \epsilon$ $\left(\mu_{3}, \mu_{4}\right)$ which means coexistence of spotted and stripe patterns will emerge. When the time is small, the differences of patterns between Figs. 4 and 8 are not distinct. However, as time increases, spotted and stripe patterns spring up, which confirms the theoretical results in amplitude equation.

We show the spatial pattern of vegetation at 500,000 iterations with $S=64$ in Fig. 9. These parameters set is corresponding to that $\mu=0.46573429>\mu_{4}$, which means stripe patterns will emerge. From numerical simulations, we can draw a conclusion: numerical results correspond perfectly to our theoretical findings.

Figure 10 illustrate wavelength of vegetation pattern as a function of parameters S and B. Figure 10(a) shows dependence on the rainfall with fixed plant morality. The wavelength becomes smaller as rainfall increases. On the other hand, Fig. 10(b) shows that the wavelength becomes larger as plant morality increases. 


\section{Discussion and conclusion}

In this paper, we have investigated the vegetation pattern in an arid flat environment by giving the conditions on the emergence of spatial patterns. By standard multiple-scale analysis, we give the pattern selection of vegetation model. Furthermore, we show the relationship between parameters ( $\mathrm{S}$ and $\mathrm{B}$ ) and wavelength of the spatial pattern by using dispersion relation. It can be found from this paper, vegetation in an arid flat environment could have rich pattern structures, including spotted, mixed, and stripe patterns.

Rainfall may be one of the important factors in the vegetation formation. We found that when rainfall is

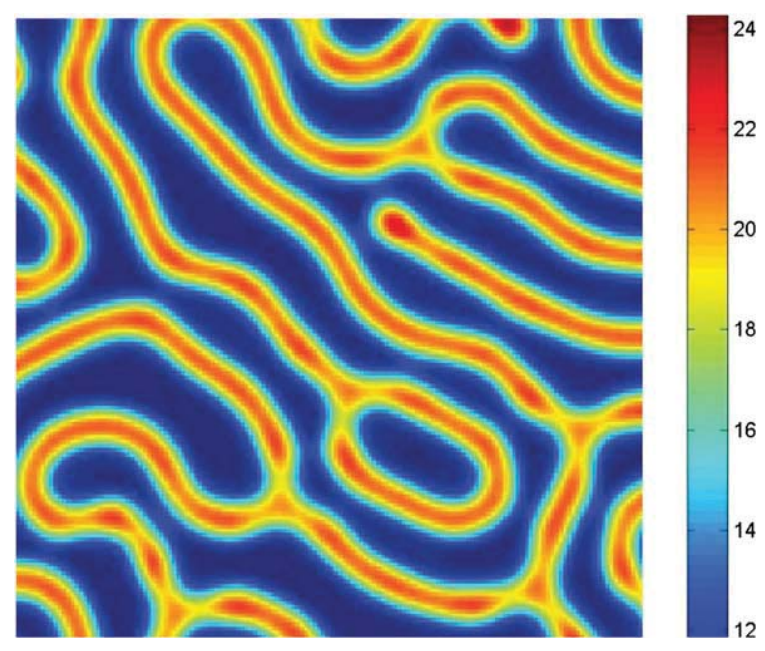

Fig. 9 Stripe pattern of vegetation at 500,000 iterations with $S=64, D=1,000$ and $B=3$ (Color figure online)

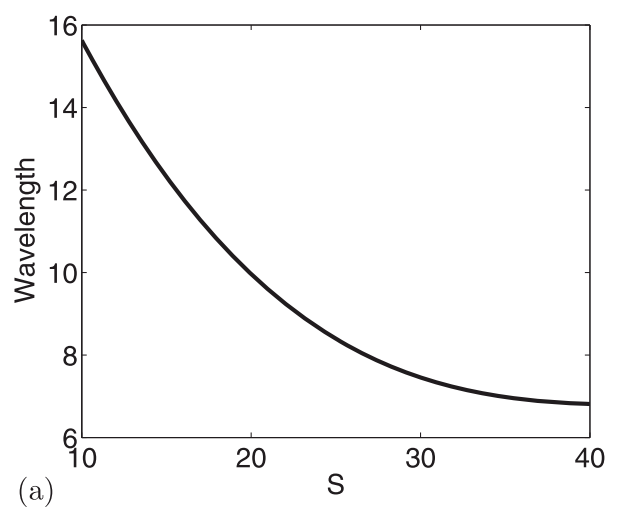

small, the system (2a), (2b) has spotted pattern. As it increases, the density of spotted pattern will increase (cf. Figs. 3 and 6). However, when it is large enough, spotted and stripe pattern emerge with lower density. It was also found that spatial pattern may be a warning signal for the onset of desertification [21, 37]. And we check that when rainfall is small enough, there is no vegetation pattern. In a word, rainfall plays an important on vegetation patterns.

In [23, 24], model (1a), (1b) is investigated in detail and it is found that there are stripe patterns. In the present paper, we found there are spotted patterns in the arid flat environment instead of downhill. In [22], they obtain the condition for emergent patterns. However, the methods are different from ours. In the Negev desert, it was found that there was a spot-like vegetation pattern [38], which is consistent with our theoretical findings. Furthermore, bistable systems can give rise to regular spatial patterns, which needs deep investigation on model (2a), (2b). Moreover, natural environments are random environments, and thus noise should be included in vegetation models. We need to check whether pattern transition can emerge in vegetation models with noise in the future work.

Acknowledgements The research was partially supported by the National Natural Science Foundation of China under Grant No. 11147015, Natural Science Foundation of Shan'Xi Province Grant Nos. 2012021002-1 and 2012011002-2, the opening foundation of the Institute of Information Economy, Hangzhou Normal University, Grant No. PD12001003002003, International and Technical Cooperation Project of Shanxi Province (2010081005) and Graduate Students' Excellent Innovative Item of Shanxi Province No. 20113107.

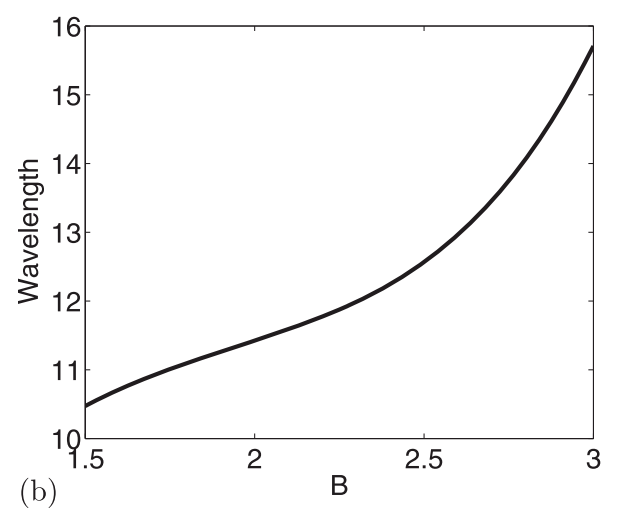

Fig. 10 This figure shows the parameters dependence of wavelength. (a) $D=1,000$ and $B=3$; (b) $D=1,000$ and $S=10$ 


\section{References}

1. Klausmeier, C.A.: Regular and irregular patterns in semiarid vegetation. Science 284, 1826-1828 (1999)

2. Rietkerk, M., van de Koppel, J.: Regular pattern formation in real ecosystems. Trends Ecol. Evol. 23, 169-175 (2007)

3. Couteron, P., Lejeune, O.: Periodic spotted patterns in semiarid vegetation explained by a propagation-inhibition model. J. Ecol. 89, 616-628 (2001)

4. HilleRisLambers, R., Rietkerk, M., van den Bosch, F., Prins, H.H.T., de Kroon, H.: Vegetation pattern formation in semiarid grazing systems. Ecology 82, 50-61 (2001)

5. von Hardenberg, J., Meron, E., Shachak, M., Zarmi, Y.: Diversity of vegetation patterns and desertification. Phys. Rev. Lett. 87, 198101 (2001)

6. Shnerb, N.M., Sara, P., Lavee, H., Solomon, S.: Reactive glass and vegetation patterns. Phys. Rev. Lett. 90, 038101 (2003)

7. Barbier, N., Couteron, P., Lejoly, J., Deblauwe, V., Lejeune, O.: Self-organized vegetation patterning as a fingerprint of climate and human impact on semi-arid ecosystems. J. Ecol. 94, 537-547 (2006)

8. Ursino, N., Rulli, M.C.: Combined effect of fire and water scarcity on vegetation patterns in arid lands. Ecol. Model. 221, 2353-2362 (2010)

9. Kefi, S., Rietkerk, M., Alados, C.L., Pueyo, Y., Papanastasis, V.P., ElAich, A., de Ruiter, P.C.: Spatial vegetation patterns and imminent desertification in Mediterranean arid ecosystems. Nature 449, 213-217 (2007)

10. Scheffer, M., Bascompte, J., Brock, W.A., Brovkin, V., Carpenter, S.R., Dakos, V., Held, H., van Nes, E.H., Rietkerk, M., Sugihara, G.: Early-warning signals for critical transitions. Nature 461, 53-59 (2009)

11. Lade, S.J., Gross, T.: Early warning signals for critical transitions: a generalized modeling approach. PLoS Comput. Biol. 8, e1002360 (2012)

12. Marinov, K., Wang, T., Yang, Y.: On a vegetation pattern formation model governed by a nonlinear parabolic system. Nonlinear Anal., Real World Appl. 14, 507-525 (2013)

13. Ursino, N.: Above and below ground biomass patterns in arid lands. Ecol. Model. 220, 1411-1418 (2009)

14. Kefi, S., Eppinga, M.B., de Ruiter, P.C., Rietkerk, M.: Bistability and regular spatial patterns in arid ecosystems. Theor. Ecol. 3, 257-269 (2010)

15. Sheffer, E., von Hardenberg, J., Yizhaq, H., Shachak, M., Meron, E.: Emerged or imposed: a theory on the role of physical templates and self-organisation for vegetation patchiness. Ecol. Lett. 16, 127-139 (2012)

16. Sherratt, J.A.: History-dependent patterns of whole ecosystems. Ecol. Complex. 14, 8-20 (2013)

17. Borgogno, F., D’Odorico, P., Laio, F., Ridolfi, L.: Mathematical models of vegetation pattern formation in ecohydrology. Rev. Geophys. 47, RG1005 (2009)

18. Ouyang, Q.: Pattern Formation in Reaction-Diffusion Systems. Shanghai Sci-Tech Education Publishing House, Shanghai (2000)
19. Peña, B., Pérez-García, C.: Stability of turing patterns in the Brusselator model. Phys. Rev. E 64, 056213 (2001)

20. Rietkerk, M., Boerlijst, M.C., van Langevelde, F., HilleRisLambers, R., van de Koppel, J., Kumar, L., Prins, H.H.T., de Roos, A.M.: Self-organization of vegetation in arid ecosystems. Am. Nat. 160, 524-530 (2002)

21. Rietkerk, M., Decker, S.C., de Ruiter, P.C., van de Koppel, J.: Self-organized patchiness and catastrophic shift in ecosystems. Science 305, 1926-1929 (2004)

22. Kealy, B.J., Wollkind, D.J.: A nonlinear stability analysis of vegetative Turing pattern formation for an interactiondiffusion plant-surfacewater model system in an arid flat environment. Bull. Math. Biol. 74, 803-833 (2012)

23. Sherratt, J.A.: An analysis of vegetative stripe formation in semi-arid landscape. J. Math. Biol. 51, 183-197 (2005)

24. Sherratt, J.A., Lord, G.J.: Nonlinear dynamics and pattern bifurcations in a model for vegetation stripes in semi-arid environments. Theor. Popul. Biol. 71, 1-11 (2007)

25. Dufiet, V., Boissonade, J.: Dynamics of Turing pattern monolayers close to onset. Phys. Rev. E 53, 4883-4892 (1996)

26. Wang, B., Wang, A.-L., Liu, Y.-J., Liu, Z.-H.: Analysis of a spatial predator-prey model with delay. Nonlinear Dyn. 62, 601-608 (2010)

27. Herrmann, H.-J.: Pattern formation of dunes. Nonlinear Dyn. 44, 315-317 (2006)

28. Sun, G.-Q., Zhang, G., Jin, Z.: Predator cannibalism can give rise to regular spatial pattern in a predator-prey system. Nonlinear Dyn. 58, 75-84 (2009)

29. Sun, G.-Q., Jin, Z., Li, L., Li, B.-L.: Self-organized wave pattern in a predator-prey model. Nonlinear Dyn. 60, 265275 (2010)

30. Pinto, C.M.A., Machado, J.T.A.: Fractional central pattern generators for bipedal locomotion. Nonlinear Dyn. 62, 27 37 (2010)

31. Fasani, S., Rinaldi, S.: Remarks on cannibalism and pattern formation in spatially extended prey-predator systems. Nonlinear Dyn. 62, 2543-2548 (2012)

32. Cross, M.C., Hohenberg, P.C.: Pattern formation outside of equilibrium. Rev. Mod. Phys. 65, 851-1112 (1993)

33. Sun, G.-Q., Jin, Z., Tan, Q.: Measurement of selforganization in arid ecosystems. J. Biol. Syst. 18, 495-508 (2010)

34. Gunaratne, G., Ouyang, Q., Swinney, H.: Pattern formation in the presence of symmetries. Phys. Rev. E 50, 2802 (1994)

35. Manneville, P.: Dissipative Structures and Weak Turbulence. Academic Press, San Diego (2000)

36. Zhang, X.-C., Sun, G.-Q., Jin, Z.: Spatial dynamics in a predator-prey model with Beddington-DeAngelis functional response. Phys. Rev. E 85, 021924 (2012)

37. Scheffer, M., Carpenter, S., Foley, J.A., Folke, C., Walker, B.: Catastrophic shifts in ecosystems. Nature 413, 591-596 (2001)

38. Meron, E., Yizhaq, H., Gilad, E.: Localized structures in dryland vegetation: forms and functions. Chaos 17, 037109 (2007) 